\title{
Beam transport line with scaling fixed field alternating gradient type magnets
}

\author{
Shinji Machida* \\ ASTeC, STFC Rutherford Appleton Laboratory, Harwell Science and Innovation Campus, Didcot, Oxfordshire, OX11 0QX, \\ United Kingdom \\ Richard Fenning \\ School of Engineering and Design, Brunel University, Kingston Lane, Uxbridge, Middlesex, UB8 3PH, United Kingdom
}

(Received 16 January 2009; published 11 August 2010)

\begin{abstract}
A scaling fixed field alternating gradient (FFAG) accelerator provides large momentum acceptance despite the fact that magnetic guiding fields are constant in time. Optical functions are identical over the large momentum range as well. We have designed a straight beam transport line (BTL) using a scaling FFAG type magnet which has a field profile of $y^{k}$, where $y$ is the horizontal coordinate and $k$ is the magnetic field index. This FFAG-BTL has very large momentum acceptance and optical functions that, practically speaking, do not depend on momentum. We also designed a dispersion suppressor at the end of the line by combining unit cells with a different field index $k$ so that the momentum dependence of orbit location should be eliminated at the exit. An obvious application of this design is the BTL after an FFAG accelerator to a patient in a hadron therapy facility or to a target in general. This could be an alternative to the conventional BTL with solenoids or quadrupoles because of the strong focusing nature of a quadrupole and the large momentum acceptance like a solenoid.
\end{abstract}

DOI: 10.1103/PhysRevSTAB.13.084001

PACS numbers: 29.27. $-\mathrm{a}, 41.85 .-\mathrm{p}, 87.56 .-\mathrm{v}$

\section{INTRODUCTION}

An ordinary synchrotron with an alternating gradient focusing structure cannot transport a beam with large momentum spread because the sizable dispersion function causes a significant shift in the closed orbit of the off momentum particle and it hits a vacuum chamber. The chromaticity cannot be zero for a large momentum range due to the inherent nonlinearity; as a result, the focusing structure will lose stability beyond some momentum range. By comparison, the scaling fixed field alternating gradient (FFAG) accelerator keeps the closed-orbit shift depending on particle momentum very small. The focal length is scaled with only the average orbit radius so that the tune is independent of momentum. This can be done with a magnetic field profile which has steeper gradient toward a large orbit radius. Although strong nonlinearities are inherently introduced, it has been shown that large momentum aperture as well as large transverse acceptance are obtained [1,2].

A FFAG accelerator can be operated with high repetition frequency because of the constant magnetic field. Neither the ramping of pulsed magnets nor the synchronization between the magnetic field and the rf frequency is necessary because the orbit is allowed to move. For example, the proof of principle proton FFAG demonstrated a $1 \mathrm{kHz}$ operation, which is impossible with an ordinary synchro-

*shinji.machida@stfc.ac.uk tron [3]. The repetition frequency is only limited by the available rf voltage and its frequency modulation. For a proton driver, without increasing the number of particles per bunch, a high current operation becomes possible. For a hadron therapy accelerator, a high repetition operation makes the spot scanning technique practical. An FFAG has the potential to deliver a beam with different momentum in a pulse by pulse mode.

Since such an accelerator has the capability of fast switching beam momentum, the following beam transport line (BTL) should have a large momentum acceptance as well. A BTL with a large momentum acceptance is also desirable as a dump line of a high intensity synchrotron where a beam that has tripped during acceleration goes through. Although the idea of using the scaling FFAG optics, where the closed-orbit shift depending on particle momentum is very small and the chromaticity is zero for a large momentum range, for a BTL has been around for years, there has been no design up to now. A BTL design with the nonscaling FFAG optics, for example, by Trbojevic [4], is limited to certain types of geometry. This paper will describe a way of designing a BTL using the scaling FFAG optics. In Sec. II, we will show a model of a magnet which is the essential element of the FFAGBTL. We will also show a unit cell as the minimum focusing component of the BTL and study its tunability, acceptance, and tolerances against several machine errors. In Sec. III, we will describe a way of obtaining the dispersion suppression. 


\section{BEAM TRANSPORT DESIGN}

\section{A. Rectangular scaling type magnet}

A scaling FFAG type magnet for a BTL can be made as follows. In a circular scaling FFAG accelerator, the field profile in cylindrical coordinates, which satisfies the scaling optics [5,6], is described as

$$
B_{z}(r, \theta)=B_{z 0}\left(\frac{r}{r_{0}}\right)^{k} F(\theta),
$$

where $B_{z}(r, \theta)$ is the vertical magnetic field, $B_{z 0}$ is the reference field at $r_{0}$, which is the reference radius measured from the machine center, $k$ is the field index, and $F(\theta)$ is a periodic function around the ring. The magnets in a scaling FFAG are wedge shaped with edges perpendicular to the arc and magnetic field lines that follow the arc.

In a BTL, on the other hand, there is no machine center. By analogy to the magnet of a scaling FFAG, however, we define a magnet for the FFAG-BTL with the field profile

$$
B_{z}(x, y)=B_{z 0}\left(\frac{y_{0}+y}{y_{0}}\right)^{k} F(x),
$$

where $x, y$, and $z$ are Cartesian coordinates, with $x$ longitudinal, $y$ horizontal, and $z$ vertical. In fact, in the limit of large $r_{0}$ of Eq. (1), an arc length of a FFAG ring becomes a straight line, which implies Eq. (2) is a reasonable assumption with large $y_{0}$. The field strength $B_{z 0}$ is adjusted so that a beam goes through near the $x$ axis, which will be later shown possible. In order to keep the focusing strength at the lower order unchanged, $k$ is increased by the same amount when $y_{0}$ is increased. As the expansion of the field shows,

$$
\begin{aligned}
& B_{z 0}\left(\frac{y_{0}+y}{y_{0}}\right)^{k} \\
& =B_{z 0}\left(1+\sum_{n=1} \frac{1}{n !} \frac{k(k-1) \cdots(k-n+1)}{y_{0}^{n}} y^{n}\right),
\end{aligned}
$$

the first quadrupole term is proportional to $k / y_{0}$. As for a different form in the limit of large $r_{0}$ [7], see Appendix A for more discussions.

Notice that the magnet has a rectangular shape and the constant field lines are straight along the BTL. The function $F(x)$ which describes the longitudinal position dependence of the fields is unity in the core of the magnets, zero far from the magnets, and has an Enge function falloff in between, which is described by

$$
F(x)=\frac{1}{1+\exp \left[\sum_{i=0}^{5} C_{i}(x / g)^{i}\right]},
$$

where $C_{i}$ are the Enge coefficients and $g$ is the gap of the magnet [8]. We assumed that $g$ is constant and independent of transverse coordinate. The overlap of fields from adjacent magnets is included as taking a summation of both.
Since Eq. (2) only describes the fields at the median plane, the off plane fields are described as a series expansion,

$$
\begin{aligned}
& B_{x}(x, y, z)=\sum_{i=0} B_{x, i}(x, y) z^{i} \\
& B_{y}(x, y, z)=\sum_{i=0} B_{y, i}(x, y) z^{i} \\
& B_{z}(x, y, z)=\sum_{i=0} B_{z, i}(x, y) z^{i} .
\end{aligned}
$$

We keep the terms up to $i=4$ and truncate the rest. The coefficients are obtained iteratively by imposing Maxwell's equations.

\section{B. Orbit and optics of unit cell}

In the following optics calculation and particle tracking, we use s-code $[9,10]$. In $s$-code, all the elements including different orders of multipoles and rf cavities are represented as a collection of thin lenses, which include end field regions. At each thin lens, momenta are updated according to the magnetic field strength. A particle goes straight between thin lenses.

In order to make an alternating gradient focusing, magnets of this type with alternating signs are aligned sequentially along a straight line. As the simplest example, we considered the FDDF configuration, where $F$ is a horizontally focusing magnet and $D$ is defocusing. This can be also regarded as a singlet $F D$ focusing structure starting from the center of the $F$ magnet. In this configuration, the orbit at entrance and at exit becomes perpendicular to the magnet faces.

Using the parameters listed in Table I, the orbit and optics with a periodic boundary condition were calculated. We took a reference momentum and momentum range according to the typical values of a hadron therapy facility. From the practical fabrication point of view, we only used a few lower order multipoles in Eq. (3). Figure 1 shows how many multipoles were necessary to keep phase advance per unit cell with a periodic boundary condition (hereafter, cell

TABLE I. Parameters of a FDDF cell.

\begin{tabular}{ll}
\hline \hline Focusing structure & $F D D F$ \\
Magnet length & $0.2 \mathrm{~m}$ \\
Drift length & $0.2 \mathrm{~m}$ \\
Cell length & $1.6 \mathrm{~m}$ \\
Reference radius $y_{0}$ & $1 \times 10^{6} \mathrm{~m}$ \\
(Field index $k$ ) $/ y_{0}$ & $5 \mathrm{~m}^{-1}$ \\
Reference momentum & $0.5 \mathrm{GeV} / c$ \\
Momentum range & 0.25 to $0.75 \mathrm{GeV} / c$ \\
$B_{z 0}$ at $F$ & $2.0 \mathrm{~T}$ \\
$B_{z 0}$ at $D$ & $-3.0 \mathrm{~T}$ \\
Enge coefficients & $0.1455,2.2670,-0.6395$, \\
$\quad C_{i}(i=0,5)$ & $1.1558,0,0$ \\
Gap of magnet $g$ & $0.15 \mathrm{~m}$ \\
\hline \hline
\end{tabular}



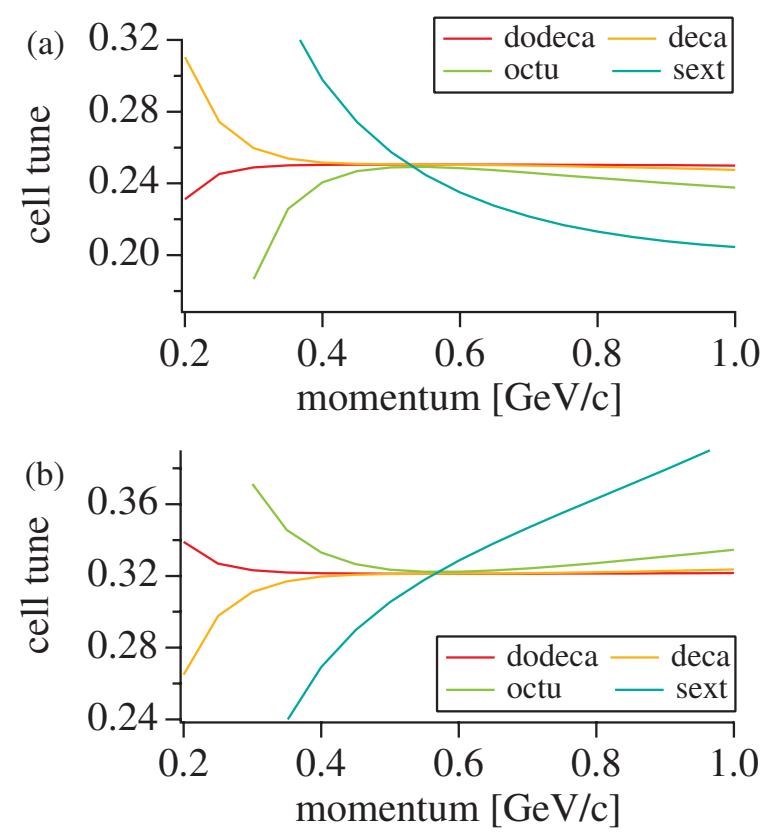

FIG. 1. (Color) Cell tune with momentum: (a) horizontal, (b) vertical. The terms dodeca, deca, octa, and sext indicate that the maximum order of the expansion is dodecapole, decapole, octapole, and sextupole, respectively.

tune in the unit of $2 \pi$ ) flat over the momentum range. It shows that the multipole field up to decapole ( $y^{4}$ term) gives fairly good approximation of the $r^{k}$ field profile. The magnetic field profile is shown in Fig. 2. A practical magnet design would be the one in Ref. [11]. There is an independent coil for each multipole. We can shape the field profile by adjusting current to each coil. In the following, all the calculations were done with this multipole based field profile instead of the field of Eq. (2).

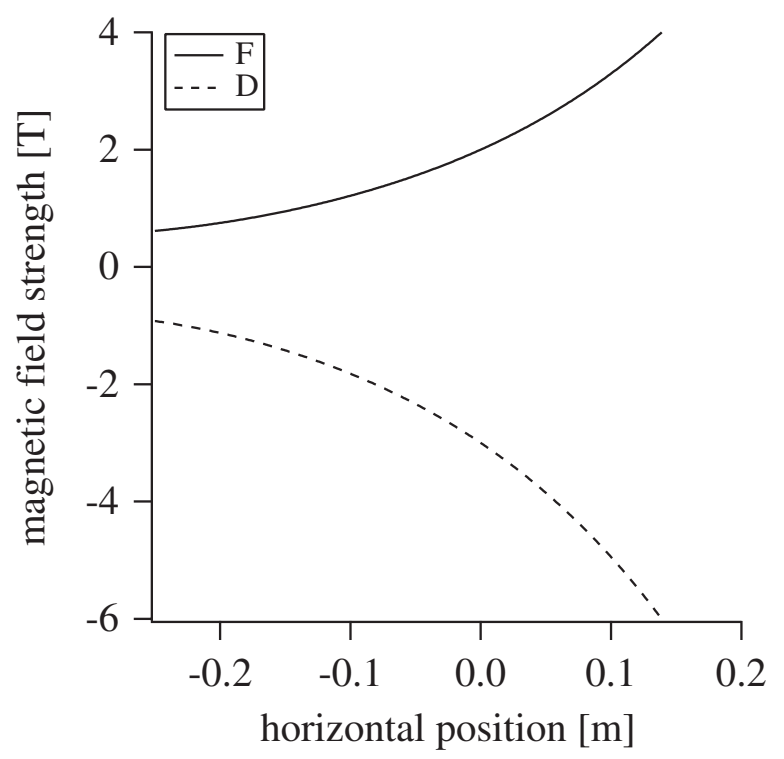

FIG. 2. Field profile of the magnet made from multipoles up to decapole.

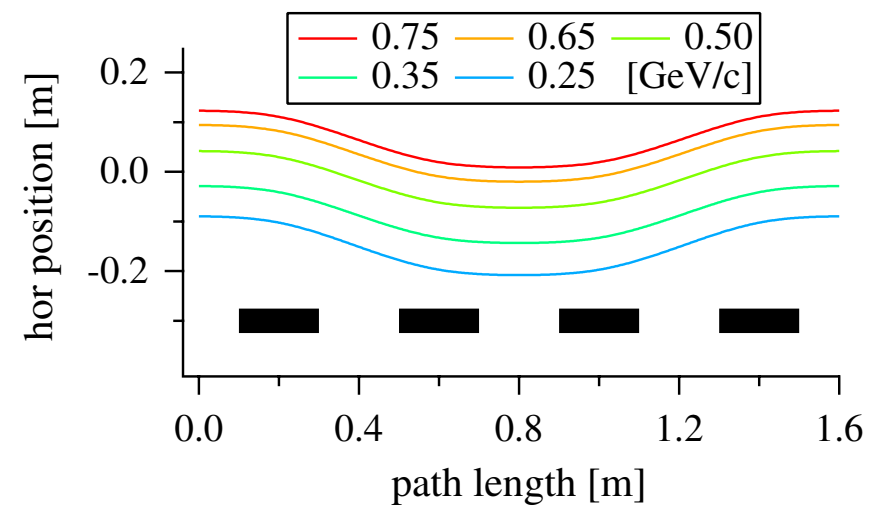

FIG. 3. (Color) Different momentum orbits in a unit cell which satisfies the periodic boundary condition. Rectangles at the bottom show the position of FDDF magnets.

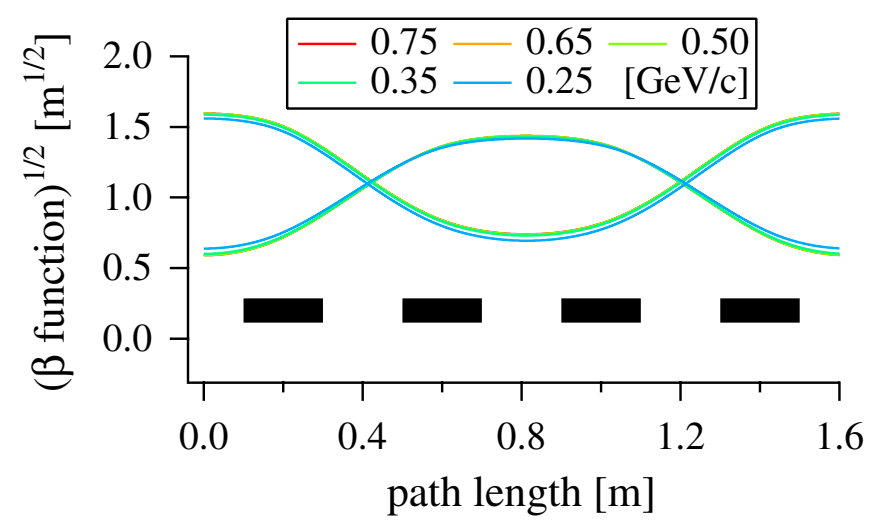

FIG. 4. (Color) Beta functions with different momenta in a unit cell which satisfies the periodic boundary condition. Rectangles at the bottom show the position of FDDF magnets.

Figure 3 shows that the shape of the orbits for different momenta are nearly identical and only slightly shifted. Figure 4 shows the beta function for different momenta. They are almost on top of each other.

\section{Tunability}

The cell tune can be adjusted by the field index $k$, or more precisely speaking $k / y_{0}$, and the ratio of field strength at $F$ and $D$ as in an ordinary scaling FFAG accelerator. The cell tune as a function of the field index and the ratio of field strength $-B_{z 0, D} / B_{z 0, F}$ is calculated with the magnet length and the lattice layout fixed. Figure 5 shows the cell tune space which can be covered with those variables.

\section{Beam transport line}

A BTL is made up of many unit cells. We took, as an example, 100 cells and studied the acceptance. The tolerances against several machine errors: alignment, field strength, and field shape were also investigated. 


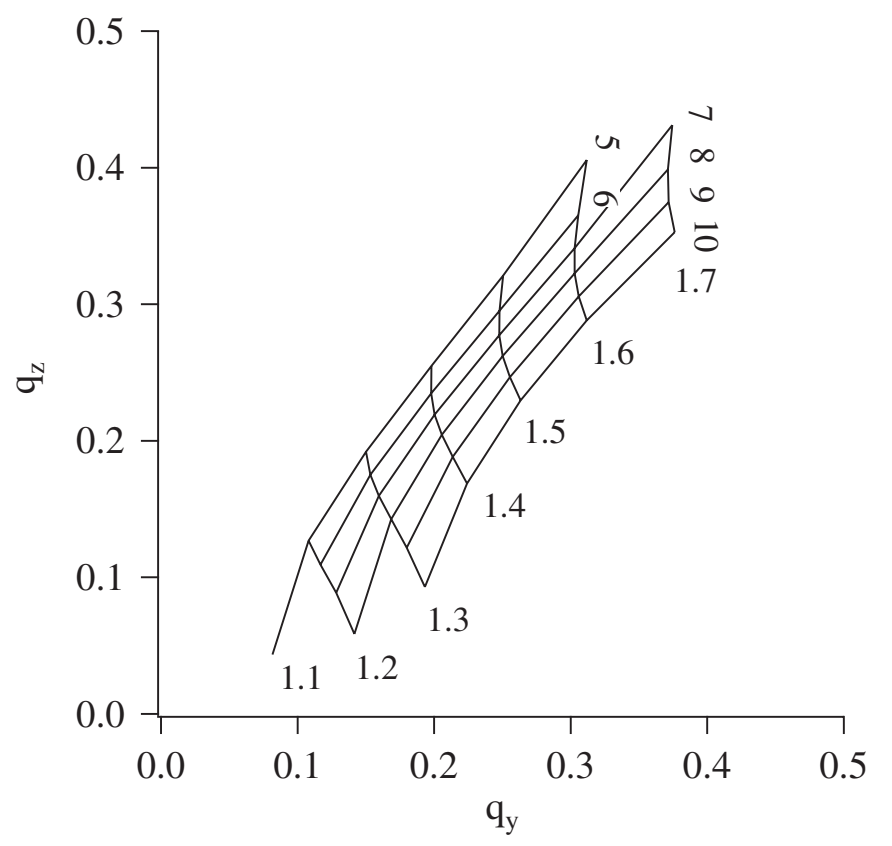

FIG. 5. Phase advance $/ 2 \pi$ in a unit cell with different (field index $k$ ) $/ y_{0}$ and ratio of field strength at $F$ and $D$. Upright numbers indicate the ratio of the field strength, $-B_{z 0, D} / B_{z 0, F}$ and vertically aligned numbers indicate the (field index $k$ ) $/ y_{0}$.

Asymmetry of the field profile in the horizontal direction shown in Fig. 2 increases the restoring force on one side and decreases it on the other. When the particle amplitude exceeds a certain value, the restoring force is not enough and a particle does not come back to the beam axis. Even if there is enough restoring force, nonlinearity coming from the field profile distorts beam emittance.

At the reference momentum of $0.5 \mathrm{GeV} / c$, the largest amplitude particle which could be transported in the FFAG-BTL was calculated. A set of five initial phase space positions with the same horizontal amplitude was chosen uniformly in the azimuthal direction in horizontal phase space. At each horizontal phase space position, five particles are allocated which are also uniformly distributed in the azimuthal direction in vertical phase space. Therefore, each particle has the same horizontal and vertical amplitude, but the phase in transverse phase space is different. The total number of particles is 25 . If the amplitude of any one of the particles started growing monotonically rather than oscillations, we considered that the initial amplitude to be outside the aperture. We chose operating points at the grids of Fig. 5. Table II shows the acceptance in terms of unnormalized value. The acceptance is much larger than beam emittance for typical proton beam such as $10 \pi \mathrm{mm}$ mrad (unnormalized).

At the nominal operating point with the (field index $k$ ) $/ y_{0}=5$ and the ratio $B_{z 0, D} / B_{z 0, F}=1.5$, the edge of a $10 \pi \mathrm{mm}$ mrad unnormalized emittance beam was tracked for 100 cells. Phase space at the end of
TABLE II. Acceptance of the FFAG-BTL [ $\pi \mathrm{mm}$ mrad $]$ unnormalized.

\begin{tabular}{lrrrrrr}
\hline \hline & \multicolumn{6}{c}{ (Field index $k) / y_{0}$} \\
& 5 & 6 & 7 & 8 & 9 & 10 \\
$-B_{z 0, D} / B_{z 0, F}$ & & & & & & \\
1.1 & 1420 & $\ldots$ & $\ldots$ & $\ldots$ & $\ldots$ & $\ldots$ \\
1.2 & 1820 & 1240 & 700 & 120 & $\ldots$ & $\ldots$ \\
1.3 & 1610 & 1040 & 940 & 570 & 310 & 120 \\
1.4 & 800 & 470 & 660 & 190 & 220 & 270 \\
1.5 & 80 & 290 & 170 & 50 & 40 & 100 \\
1.6 & 100 & 20 & 10 & 130 & 110 & 70 \\
1.7 & $\cdots$ & $\ldots$ & 30 & 30 & 20 & 20 \\
\hline \hline
\end{tabular}

the BTL is shown in Fig. 6. In this case, 18 phase space positions with the same amplitude in horizontal direction have 18 particles in vertical direction. Not much distortion of beam emittance is observed except a little square shape in horizontal phase space.
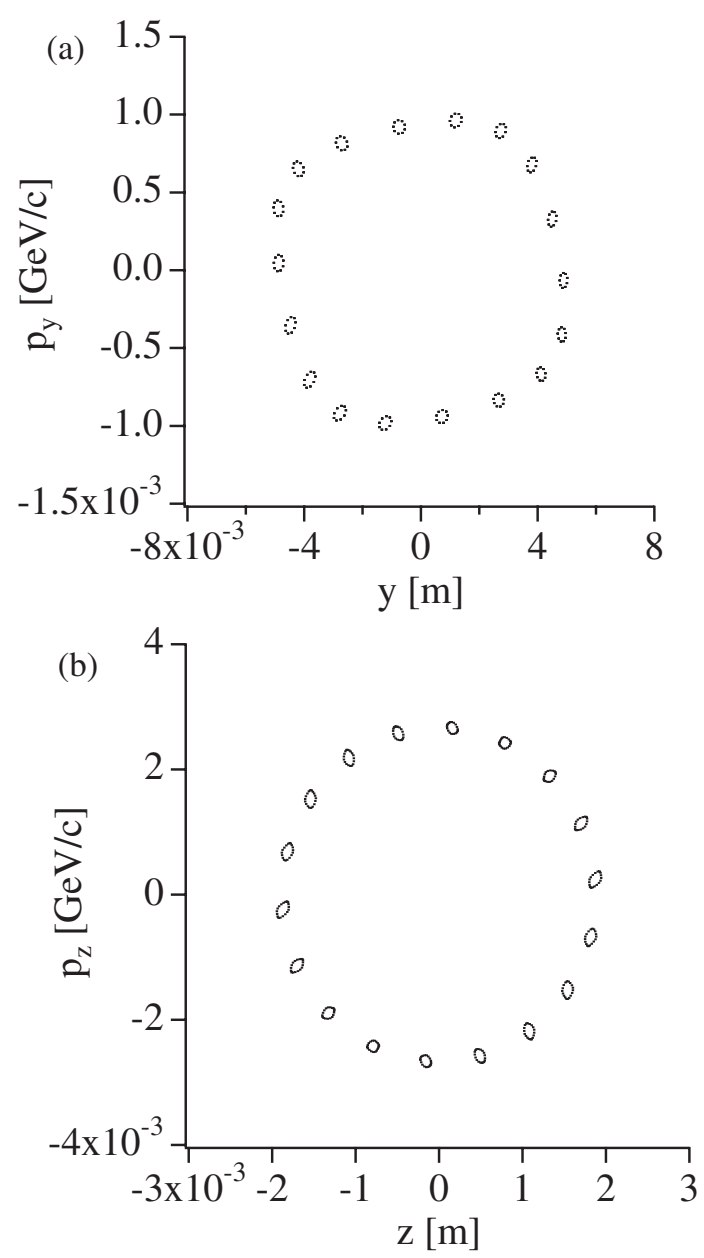

FIG. 6. (a) Horizontal and (b) vertical edge of a beam after going through 100 cells. 18 phase space positions with the amplitude of $10 \pi \mathrm{mm}$ mrad in one direction has 18 particles in the other direction. 
TABLE III. Maximum orbit distortion due to $0.1 \mathrm{~mm}$ (sigma) alignment error or $0.1 \%$ (sigma) strength and shape errors. The data is consistent with a linear relation between the distortion and errors as shown in Fig. 7. Strength and shape errors do not cause orbit distortion in the vertical direction.

\begin{tabular}{lccc}
\hline \hline Error & $\begin{array}{c}\text { Momentum } \\
{[\mathrm{GeV} / c]}\end{array}$ & $\begin{array}{c}\text { Horizontal } \\
{[\mathrm{mm}]}\end{array}$ & $\begin{array}{c}\text { Vertical } \\
{[\mathrm{mm}]}\end{array}$ \\
\hline Alignment & 0.25 & $5.2 \pm 1.9$ & $4.5 \pm 1.5$ \\
& 0.5 & $5.4 \pm 1.9$ & $4.8 \pm 1.7$ \\
Strength & 0.75 & $5 \pm 2$ & $4.8 \pm 1.7$ \\
& 0.25 & $9 \pm 3$ & $\ldots$ \\
Shape & 0.5 & $9 \pm 3$ & $\ldots$ \\
& 0.75 & $9 \pm 3$ & $\ldots$ \\
& 0.25 & $6 \pm 2$ & $\ldots$ \\
\hline \hline
\end{tabular}

In order to see tolerances against several machine errors, we introduced three different kinds of error. One was a magnet alignment error. Another was an error in the field strength by introducing some fluctuations in $B_{z 0}$. The third was an error in the field shape by introducing fluctuations in $k$. We provided 501 different patterns for each error assuming that the error distribution was Gaussian with two sigma cut off. The sigma value for each error was varied from 0 to $0.2 \mathrm{~mm}$ for an alignment error and from $0 \%$ to $0.2 \%$ for both field strength and field shape errors. $B_{z 0}$ at $D$ for field shape error is change from -3.0 to $-2.8 \mathrm{~T}$ to avoid nearby $4 q_{y}=1$ resonance where $q_{y}$ is horizontal cell tune. The maximum orbit distortion and the growth of square root of beta functions were then calculated for each error pattern and normalized by the magnitude of the error. The whole procedure was repeated with several momenta. Tables III and IV show the mean and the standard deviation of the results.

The tables show that the maximum orbit distortion is a few $\mathrm{mm}$ to $10 \mathrm{~mm}$ and the maximum growth of square root

TABLE IV. Maximum growth of square root of beta functions due to $0.1 \mathrm{~mm}$ (sigma) alignment error or $0.1 \%$ (sigma) strength and shape errors. The data is consistent with a linear relation between the growth and errors as shown in Fig. 8.

\begin{tabular}{lccc}
\hline \hline Error & $\begin{array}{c}\text { Momentum } \\
{[\mathrm{GeV} / c]}\end{array}$ & $\begin{array}{c}\text { Horizontal } \\
{[\%]}\end{array}$ & $\begin{array}{c}\text { Vertical } \\
{[\%]}\end{array}$ \\
\hline Alignment & 0.25 & $6 \pm 2$ & $3.9 \pm 1.8$ \\
& 0.5 & $10 \pm 20$ & $5 \pm 3$ \\
Strength & 0.75 & $10 \pm 16$ & $5 \pm 3$ \\
& 0.25 & $5 \pm 2$ & $4 \pm 4$ \\
Shape & 0.5 & $7 \pm 4$ & $1.5 \pm 0.5$ \\
& 0.75 & $7 \pm 4$ & $1.5 \pm 0.5$ \\
& 0.25 & $5 \pm 2$ & $3.3 \pm 1.1$ \\
& 0.5 & $1.7 \pm 1.2$ & $2.3 \pm 0.7$ \\
\hline \hline
\end{tabular}

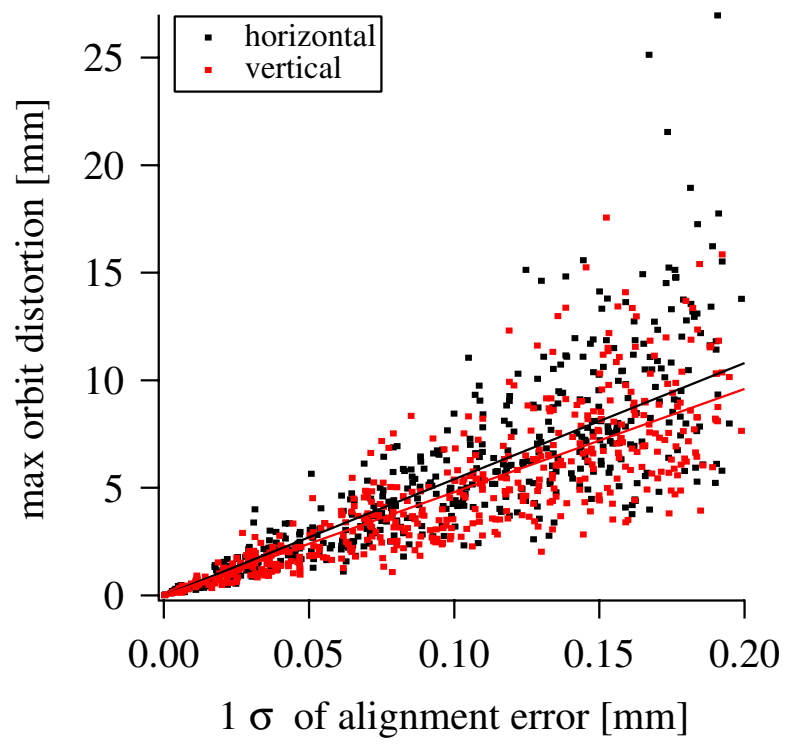

FIG. 7. (Color) Maximum orbit distortion against alignment errors. Straight lines indicate linear fit.

of beta functions is a few $\%$ to $10 \%$ with reasonable assumption of various errors [12].

\section{DISPERSION SUPPRESSOR}

In the FFAG-BTL, a particle with different momentum has a different central orbit. If we consider an application such as a BTL right after a FFAG accelerator, the orbits may be separated already at the entrance to the BTL because of the orbit separation depending on momentum in the ring. However, we want to suppress the dispersion at the end of the FFAG-BTL so that all the different momentum particles go to a single spot.

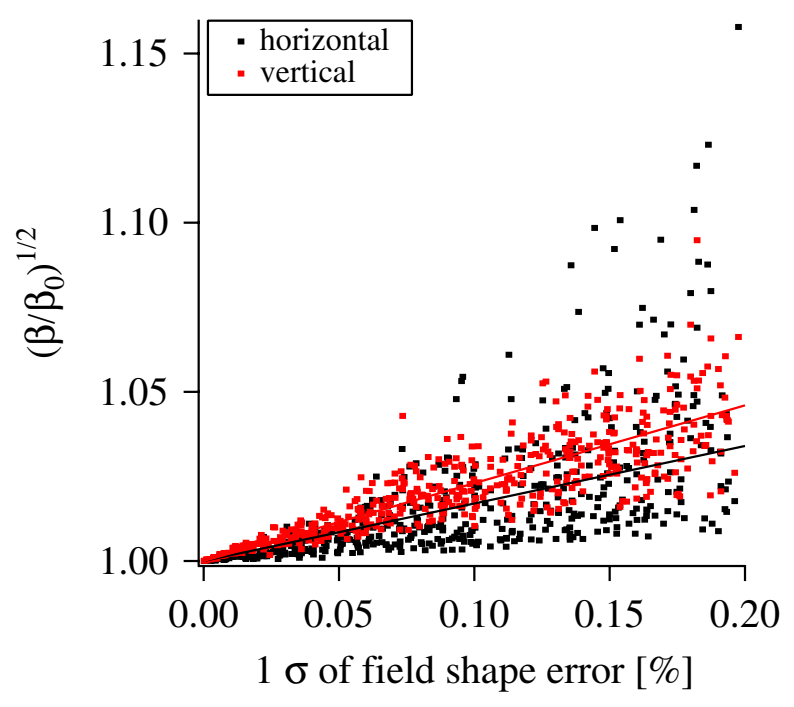

FIG. 8. (Color) Maximum growth of square root of beta functions against field shape errors. Straight lines indicate linear fit. 
We know that, if the dipole bending strength of the adjoining $-I$ section is halved, where $I$ is the identity transfer matrix, the dispersion function will be zero at the end [13]. It is called a dispersion suppressor with the missing dipole scheme. In terms of scaling FFAG optics, a dispersion suppressor can be a $-I$ section with the (field index $k$ ) $/ y_{0}$ doubled with respect to a normal cell (see Appendix B). In practice, it is easier to design a $-I$ section first. An example is two unit cells each with 90 degrees phase advance in the horizontal direction. Either the (field index $k$ ) $/ y_{0}$ or the ratio $B_{z 0, D} / B_{z 0, F}$ could be used to obtain the correct phase advance. Second, one fixes the reference momentum and calculates a periodic solution. This gives an orbit position at the ends of a cell in a dispersion suppressor. We name it the reference position. Third, one designs another unit cell with the (field index $k$ ) $/ y_{0}$ halved, which becomes a normal cell, and adjusts the field strength of $F$ and $D$ so that the reference momentum particle has the same orbit position at the ends of the cell as that of the dispersion suppressor. Finally, one connects the normal cell to the dispersion suppressor.

A reference momentum particle entering a normal cell at the reference position comes back to the reference position at the exit of the cell. Then, it enters the dispersion suppressor at the reference position and comes back to the reference position at the exit of the cell with a smaller horizontal shift within the dispersion suppressor cell. A particle with different momentum has a shifted central orbit in the normal cell. It comes back to the same horizontal position at the exit as at the entrance in the normal cell. However, it goes to the reference position once it exits the dispersion suppressor if the $-I$ condition is preserved.

In fact, the $-I$ condition is only approximately satisfied for off momentum particles because the shape of orbits in the dispersion suppressor is not similar and the phase advance strongly depends on the orbit position as shown in Fig. 9. We have looked at the transverse position at the exit of the suppressor as a function of momentum deviation

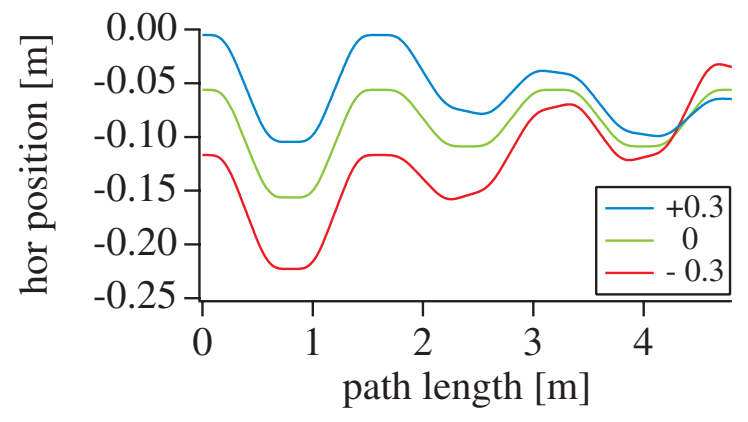

FIG. 9. (Color) Different momentum orbits in a normal cell and the dispersion suppressor. Numbers in the legend show the momentum deviation from the reference momentum, $\Delta p / p$. The normal cell is from 0 to $1.6 \mathrm{~m}$ and the dispersion suppressor section from 1.6 to $4.8 \mathrm{~m}$.

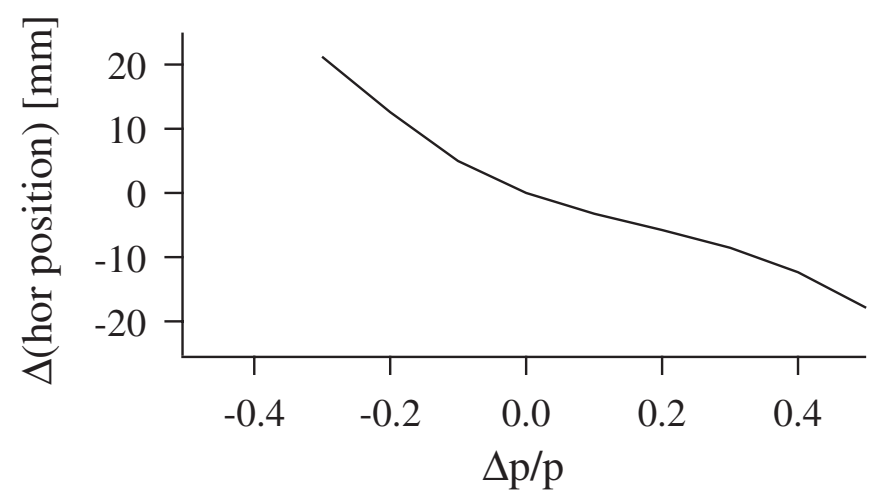

FIG. 10. Relative horizontal orbit position at the exit of the dispersion suppressor as a function of momentum deviation. Zero corresponds the orbit position of a reference momentum particle.

in Fig. 10. This shows the dispersion suppressor works only in the limited momentum range. Although FFAG-BTL itself has larger momentum acceptance, the dispersion suppressor determines the momentum range we can use. It is still large enough for many purposes such as a hadron therapy facility. Failure to meet an exact condition of $-I$ also affects the beta functions in the dispersion suppressor. Figure 11 shows the beta function for different momenta. Because of the variation of the beta functions, the beam size at the end becomes a function of momentum. The beam size at the exit of the suppressor as a function of momentum deviation is shown in Fig. 12. Within the
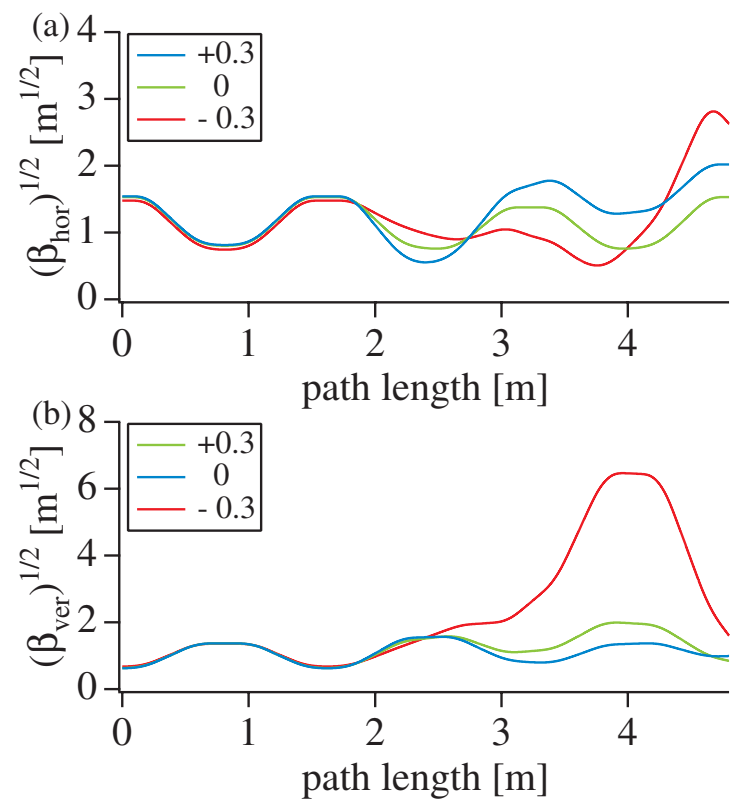

FIG. 11. (Color) (a) Horizontal and (b) vertical beta functions in a normal cell and the dispersion suppressor. Numbers in the legend show the momentum deviation from the reference momentum, $\Delta p / p$. The normal cell is from 0 to $1.6 \mathrm{~m}$ and the dispersion suppressor section from 1.6 to $4.8 \mathrm{~m}$. 

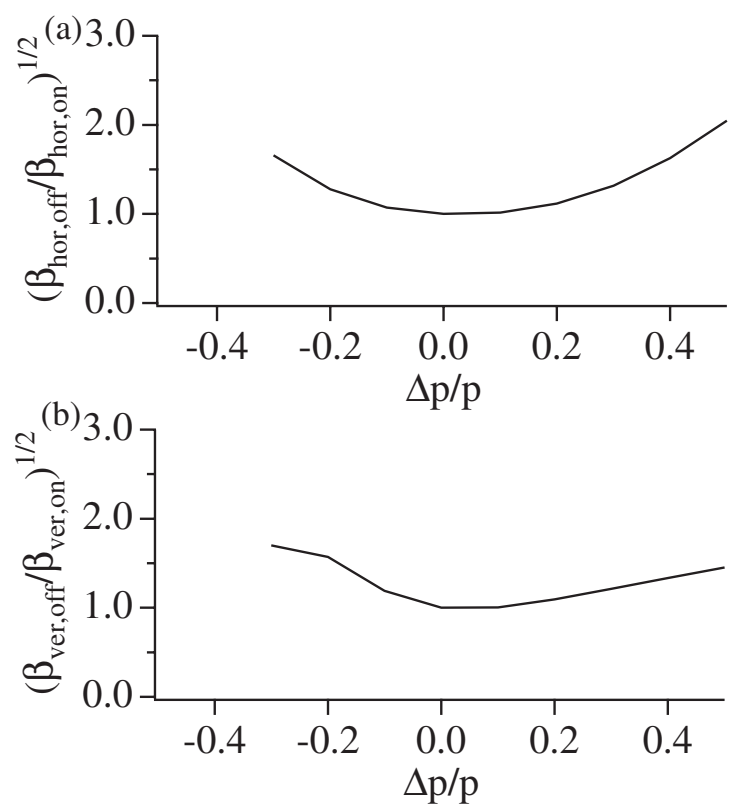

FIG. 12. (a) Horizontal and (b) vertical square root of beta functions of off-momentum beam normalized by that of the onmomentum one as a function of momentum deviation.

momentum range of $\pm 30 \%$, the beam size varies by around $\pm 30 \%$. Nevertheless, for a target or a patient, the small variation of beam size should not be a problem.

\section{SUMMARY}

With a scaling FFAG accelerator type magnet, we have designed a beam transport line (FFAG-BTL). Although the magnetic field is constant in time, it has a large momentum acceptance. We studied the tunability of transverse focusing, transverse acceptance, and tolerance against several machine errors. We also designed a dispersion suppressor section so that the shifted orbits depending on beam momentum can be merged into a spot at the end of the line. Although the momentum range is limited over which the dispersion suppressor works reasonably well, that is around $\pm 30 \%$, it should be enough for many applications.

A possible use of the FFAG-BTL is for beam transport between a FFAG accelerator and a target or a patient downstream. With large momentum acceptance, it becomes possible to change the beam momentum pulse by pulse, which realizes the spot scanning technique when applied to a hadron therapy facility. Another use of the FFAG-BTL with a large momentum acceptance is a dump line of a high intensity synchrotron where a beam that has tripped during acceleration goes through.

\section{ACKNOWLEDGMENTS}

The work is supported by the U.K. Neutrino Factory/ Particle Physics and Astronomy Research Council (PPARC) under Contract No. 2054. We would like to thank Dr. C. R. Prior, Dr. G. H. Rees, Dr. Y. Mori, Dr. S. Ohnuma,
Dr. K. J. Peach, Dr. H. Witte, Dr. T.R. Edgecock, and Dr. A. Khan for their fruitful discussions and continuous encouragement, and Dr. S. J. Brooks and Dr. D. J. Kelliher for helpful comments on the manuscript.

\section{APPENDIX A}

Mori, Planche, and Lagrange also proposed the field profile of a straight beam transport line, which satisfied the scaling law [7]. In the straight line, the scaling law means that the orbit shape and optics functions become identical independent of beam momentum. According to the reference, the field profile should be

$$
B_{z}(x, y)=B_{z 0} \exp \left(\frac{n}{\rho} y\right) F(x),
$$

where $n=\left(\rho / B_{z}\right)\left(d B_{z} / d y\right)$ and $\rho$ is the radius of curvature. Equation (A1) is actually the form of Eq. (2) in the limit of large $y_{0}$ as shown in [14]:

$$
\begin{aligned}
\lim _{y_{0} \rightarrow \infty}\left(\frac{y_{0}+y}{y_{0}}\right)^{k} & =\lim _{y_{0} \rightarrow \infty}\left[\left(1+\frac{y}{y_{0}}\right)^{y_{0} / y}\right]^{\left(k / y_{0}\right) y} \\
& =\lim _{y_{0} \rightarrow \infty}\left[\left(1+\frac{y}{y_{0}}\right)^{y_{0} / y}\right]^{(n / \rho) y}=\exp \left(\frac{n}{\rho} y\right),
\end{aligned}
$$

where $k / y_{0}$ is replaced by $n / \rho$ before taking the limit of large $y_{0}$.

Unfortunately, the derivation of the field profile in [7] is not rigorous although the result is correct. We will show that Eq. (A1) indeed gives the scaling law in the following way.

Suppose there is a particle whose momentum is $p_{1}$ and goes through the horizontal position of $y_{1}$ and longitudinal position of $x_{1}$. According to Eq. (A1), it sees the bending and focusing strength of

$$
B_{z}\left(x_{1}, y_{1}\right)=B_{z 0} \exp \left(\frac{n}{\rho} y_{1}\right) F\left(x_{1}\right)
$$

and

$$
\frac{d B_{z}\left(x_{1}, y_{1}\right)}{d y}=\frac{n B_{z 0}}{\rho} \exp \left(\frac{n}{\rho} y_{1}\right) F\left(x_{1}\right) .
$$

Consider another particle whose momentum is $p_{2}=\alpha p_{1}$, where $\alpha$ is a constant. It has the same bending radius $\kappa$ and focusing strength $K$ at $y_{2}=y_{1}+\frac{\rho}{n} \log \alpha$ and $x_{2}=x_{1}$ because

$$
\begin{aligned}
\kappa & =\frac{p_{2}}{e B_{2}}=\frac{\alpha p_{1}}{e B_{z 0} \exp \left[n\left(y_{1}+\frac{\rho}{n} \log \alpha\right) / \rho\right]} \\
& =\frac{\alpha p_{1}}{e B_{z 0} \exp \left(\frac{n}{\rho} y_{1}\right) \exp (\log \alpha)}=\frac{p_{1}}{e B_{1}}
\end{aligned}
$$

and 


$$
K=\frac{\frac{d B_{z}\left(x_{2}, y_{2}\right)}{d y}}{B_{z}\left(x_{2}, y_{2}\right) \kappa_{2}}=\frac{\alpha \frac{d B_{z}\left(x_{1}, y_{1}\right)}{d y}}{\alpha B_{z}\left(x_{1}, y_{1}\right) \kappa_{1}}=\frac{\frac{d B_{z}\left(x_{1}, y_{1}\right)}{d y}}{B_{z}\left(x_{1}, y_{1}\right) \kappa_{1}}
$$

Namely, if the orbit and optics for a particular momentum particle exist, the other particles whose momentum is $p_{2}=$ $\alpha p_{1}$ have the identical orbit and optics at the horizontal position shifted by $\frac{\rho}{n} \log \alpha$.

The quantitative difference between Eq. (2) and Eq. (A1) can be estimated as follows. If the transverse part of both equations is expanded as Taylor series, Eq. (2) becomes

$$
\begin{aligned}
& B_{z 0}\left(\frac{y_{0}+y}{y_{0}}\right)^{k} \\
& =B_{z 0}\left(1+\sum_{n=1} \frac{1}{n !} \frac{k(k-1) \cdots(k-n+1)}{y_{0}^{n}} y^{n}\right) .
\end{aligned}
$$

On the other hand, Eq. (A1) becomes

$$
B_{z 0} \exp \left(\frac{n}{\rho} y\right)=B_{z 0} \exp \left(\frac{k}{y_{0}} y\right)=B_{z 0}\left(1+\sum_{n=1} \frac{1}{n !} \frac{k^{n}}{y_{0}^{n}} y^{n}\right)
$$

The difference between individual terms of those equations is the order of $\frac{1}{k}$ which is negligible when $k$ is large enough such as $5 \times 10^{6}$ in the example we took.

\section{APPENDIX B}

Let the $3 \times 3$ transfer matrix of the repetitive cell be

$$
R_{s}=\left(\begin{array}{ccc}
m_{11} & m_{12} & d \\
m_{21} & m_{22} & d^{\prime} \\
0 & 0 & 1
\end{array}\right)
$$

where $\left(\begin{array}{ll}m_{11} & m_{12} \\ m_{21} & m_{22}\end{array}\right)$ is the $2 \times 2$ transfer matrix for betatron motion and $\left(\begin{array}{l}d \\ d^{\prime}\end{array}\right)$ is the dispersion vector. The dispersion function can be obtained using the closed-orbit condition

$$
D(x+L)=D(x) \text { and } D^{\prime}(x+L)=D^{\prime}(x),
$$

where $L$ is the length of a repetitive period. Namely,

$$
R_{s}\left(\begin{array}{c}
D \\
D^{\prime} \\
1
\end{array}\right)=\left(\begin{array}{c}
D \\
D^{\prime} \\
1
\end{array}\right)
$$

When

$$
\left(\begin{array}{ll}
m_{11} & m_{12} \\
m_{21} & m_{22}
\end{array}\right)=\left(\begin{array}{cc}
-1 & 0 \\
0 & -1
\end{array}\right)=-I,
$$

the dispersion vector and the dispersion function satisfy

$$
D=\frac{d}{2} \quad \text { and } \quad D^{\prime}=\frac{d^{\prime}}{2}
$$

In an FFAG, from Eq. (2),

$$
\frac{y}{y_{0}}=\left(\frac{p_{0}+d p}{p_{0}}\right)^{1 /(k+1)}
$$

where $p_{0}$ is the reference momentum and $d p$ is a deviation. Taking the leading term, it becomes

$$
y=y_{0}+\frac{1}{k / y_{0}} \frac{d p}{p_{0}} .
$$

Therefore the dispersion function is inversely proportional to $k / y_{0}$.

When $k / y_{0}$ of the dispersion suppressor is doubled with respect to the normal cell, the dispersion function $D_{n}$ and its derivative at the normal cell and $D$ and its derivative at the suppressor cell are

$$
D_{n}=2 D \text { and } \quad D_{n}^{\prime}=2 D^{\prime} .
$$

Then,

$$
\left(\begin{array}{c}
D_{n} \\
D^{\prime}{ }_{n} \\
1
\end{array}\right)
$$

at the entrance of the dispersion suppressor becomes at the end

$$
\left(\begin{array}{ccc}
-1 & 0 & d \\
0 & -1 & d^{\prime} \\
0 & 0 & 1
\end{array}\right)\left(\begin{array}{c}
D_{n} \\
D^{\prime}{ }_{n} \\
1
\end{array}\right)=\left(\begin{array}{c}
-2 D+d \\
-2 D^{\prime}+d^{\prime} \\
1
\end{array}\right)=\left(\begin{array}{l}
0 \\
0 \\
1
\end{array}\right)
$$

The dispersion function and its derivative are zero after the dispersion suppressor.

[1] M. Yoshimoto, T. Adachi, M. Aiba, K. Koba, S. Machida, Y. Mori, R. Muramatsu, C. Ohmori, I. Sakai, Y. Sato, M. Sugaya, A. Takagi, R. Ueno, M. Wada, T. Yokoi, M. Yoshii, and Y. Yuasa, in Proceedings of the 19th Particle Accelerator Conference, Chicago, Illinois, 2001 (IEEE, Piscataway, NJ, 2001), p. 51 [http://www.jacow.org/].

[2] M. Yoshimoto, T. Adachi, M. Aiba,S. Machida, Y. Mori, A. Muto, R. Muramatsu, C. Ohmori, I. Sakai, Y. Sato, M. Sugaya, A. Takagi, R. Ueno, A. Yamazaki, T. Yokoi, U. Yonemura, M. Yoshii,Y. Yuasa, and K. Koba, in Proceedings of the 8th European Particle Accelerator Conference, Paris, 2002 (EPS-IGA and CERN, Geneva, 2002), p. 1320 [http://www.jacow.org/].

[3] M. Aiba, K. Koba, S. Machida, Y. Mori, R. Muramatsu, C. Ohmori,I. Sakai, Y. Sato, A. Takagi, R. Ueno, T. Yokoi, M. Yoshimoto, and Y. Yuasa, in Proceedings of the European Particle Accelerator Conference, Vienna, 2000 (EPS, Geneva, 2000), p. 581 [http://www.jacow.org/].

[4] D. Trbojevic, B. Parker, E. Keil, and A. M. Sessler, Phys. Rev. ST Accel. Beams 10, 053503 (2007).

[5] K. R. Symon, D. W. Kerst, L. W. Jones, L. J. Laslettand, and K. M. Terwillinger, Phys. Rev. 103, 1837 (1956).

[6] A.A. Kolomenskyand and A. N. Levedev, Theory of Cyclic Accelerators (North-Holland, Amsterdam, 1966), p. 337. 
[7] Y. Mori, T. Planche, and J. B. Lagrange, http://hadron. kek.jp/FFAG/FFAG08J_HP/.

[8] H. A. Enge, Focusing of Charged Particles, edited by A. Spetier (Academic Press, New York, 1967), Vol. 2, p. 203.

[9] S. Machida, ICFA Beam Dynamics Newsletter, edited by C. R. Prior (2007), Vol. 43, p. 54 [http://icfa-usa.jlab.org/ archive/newsletter.shtml/].

[10] S. Machida, Int. J. Mod. Phys. A (to be published).
[11] H. Witte, http://www.cockcroft.ac.uk/events/FFAG08/ programme.htm/.

[12] H. Witte (private communication).

[13] S. Y. Lee, Accelerator Physics (World Scientific, Singapore, 1999), p. 124.

[14] Y. Mori, T. Uesugi, T. Planche, and J. B. Lagrange, http:// nufact09.iit.edu/wg3.shtml\#wg3wedpm2/. 